\title{
Microarray analysis reveals Tmub1 as a cell cycle-associated protein in rat hepatocytes
}

\author{
HANGWEI FU ${ }^{1}$, JIANHUA XU ${ }^{1}$, JIAN CHEN ${ }^{1}$, GUANGYAO LI $^{1}$, XIAOBIAO ZHAO $^{2}$ and PING CHEN ${ }^{1}$ \\ ${ }^{1}$ Department of Hepatobiliary Surgery, Institute of Surgery Research, Daping Hospital, Third Military Medical University, \\ Chongqing 400042; ${ }^{2}$ Department of Hepatobiliary Surgery, 187 Military Hospital, Haikou, Hainan 571159, P.R. China
}

Received September 3, 2017; Accepted January 11, 2018

DOI: $10.3892 / \mathrm{mmr} .2018 .8451$

\begin{abstract}
Transmembrane and ubiquitin-like domain containing protein 1 (Tmub1), formerly known as hepatocyte odd protein shuttling (HOPS) has been recognized as a ubiquitously expressed shuttling protein that moves between the nucleus and cytoplasm in hepatocytes. Tmub1 is involved in liver regeneration and functions as a bridging protein in tumor cell proliferation. To investigate the transcriptional profile and potential biological processes affected by Tmub1 expression in normal rat hepatocytes, microarray and bioinformatics experiments were used to identify 127 mRNAs differentially expressed between Tmub1-overexpression, Tmub1-knockdown and normal BRL-3A cells (fold-change $\geq 2.5$ ). The expression levels of 17 key node genes associated with the cell cycle were confirmed by reverse transcription-quantitative polymerase chain reaction analysis. Flow cytometry, 5-Ethynyl-20-deoxyuridine, Cell Counting Kit-8 and western blotting experiments revealed the effects on the cell cycle and the inhibition of proliferation in BRL-3A cells overexpressing Tmub1. Further co-immunoprecipitation assays demonstrated that Tmub1 interacts with cyclin A2 during the cell cycle and
\end{abstract}

Correspondence to: Professor Ping Chen, Department of Hepatobiliary Surgery, Institute of Surgery Research, Daping Hospital, Third Military Medical University, 10 Changjiangzhilu Daping, Chongqing 400042, P.R. China

E-mail: chenpingdph@163.com

Dr Xiaobiao Zhao, Department of Hepatobiliary Surgery, 187 Military Hospital, 100 Longkunnanlu, Haikou, Hainan 571159, P.R. China

E-mail: xiaobiao123@sohu.com

Abbreviations: Tmub1, transmembrane and ubiquitin-like domain containing protein 1; Lv, lentiviral vectors; GO, gene ontology; KEGG, kyoto encyclopedia of genes and genomes; DAVID, database for annotation, visualization, and integrated discovery; IP, immunoprecipitation

Key words: transmembrane and ubiquitin-like domain containing protein 1, rat, microarray analysis, liver regeneration, cell cycle, cell proliferation, cyclin A2 that the overexpression of Tmub1 may postpone cyclin A2 and cyclin $\mathrm{B} 1$ degradation in the $\mathrm{M}$ phase. The results of the present study indicated that Tmubl functions as a cell proliferation inhibitor and cell cycle-associated protein.

\section{Introduction}

Recent studies in the field of liver regeneration have focused on how pattern recognition receptors and a variety of molecules are activated after partial hepatectomy $(\mathrm{PH})(1,2)$. Transmembrane and ubiquitin-like domain containing protein 1 (Tmub1), also named hepatocyte odd protein shuttling (HOPS)/DULP, is a key factor regulating liver-specific biological events, such as protein synthesis during liver regeneration, by binding to elongation factor eEF-1A (3). Tmub1 contains 3 transmembrane domains, a ubiquitin-like (UBL) domain and a nuclear export signal (NLS) that are critical during hepatocyte proliferation (4). Tmubl is upregulated in the regenerating liver (3) and is actively exported from the nucleus in dividing cells but predominantly located in the nucleus during growth arrest (5). Ubiquitylation is the major mechanism of protein degradation via the proteasome and can be regarded as a posttranslational modification (6), and some UBLs domain-containing proteins such as SUMO have demonstrated crosstalk with ubiquitylation (7). The functions of Tmub1 have been reported to be involved in a wide range of cellular processes not only in regenerating liver cells but also in other tissues. For instance, Tmub1 is involved in the IL-6-induced proliferation pathway in the liver (8), regulates locomotor activity and wakefulness by interacting with calcium modulating ligand (CAMLG) (9), and facilitates the recycling of the AMPAR subunit GluR2 to the cell surface in the mouse brain (10). Tmub1 is also an essential component of the centrosome assembly during the cell cycle (5). However, the physiological and molecular functions of Tmubl are far from being clear at the whole-gene transcriptional profiling level. The aim of this study was to investigate the influence of Tmub1 expression on the transcriptional profile and its possible roles in the cell cycle in the rat hepatocyte cell line BRL-3A. Our data showed that Tmub1 is primarily a cell cycle-related regulatory protein in rat hepatocytes.

\section{Materials and methods}

Antibodies. Specific rabbit anti-rat polyclonal antibodies to Tmub1 (no. ab180586), cyclin D1 (no. ab134175) and GAPDH 
(no. ab181602) were provided by Abcam (Cambridge, MA, UK). The rabbit anti-rat polyclonal cyclin A2 antibody (no. GTX103042) was provided by GeneTex Inc. (Irvine, CA, US). Rabbit anti-rat polyclonal cyclin B1 (no. w101760) and cyclin E1 (no. wl01072) antibodies were provided by Wanleibio Inc. (Shenyang, China). HRP-conjugated secondary goat anti-mouse (no. SA00001-1) and goat anti-rabbit (no. SA00001-2) antibodies were provided by Proteintech Inc. (Rosemont, IL, US). All the primary antibodies above were used at a 1:1,000 dilution with a secondary antibody at a 1:5,000 dilution for western blot analysis.

Cell culture and cell cycle synchronization. Normal rat hepatocyte cells (BRL-3A; Cell Bank of Chinese Academy of Sciences, Shanghai, China) were maintained in Dulbecco's modified Eagle's medium (Invitrogen; Thermo Fisher Scientific, Inc., Waltham, MA, USA) containing 10\% fetal bovine serum (Invitrogen; Thermo Fisher Scientific, Inc.), $100 \mathrm{U} / \mathrm{ml}$ penicillin, and $100 \mu \mathrm{g} / \mathrm{ml}$ streptomycin, and cultured in a humidified atmosphere of $5 \% \mathrm{CO}_{2}$ at $37^{\circ} \mathrm{C}$. For $\mathrm{G} 1$ phase synchronization, cells were grown in FBS-free medium for $48 \mathrm{~h}$. For $\mathrm{S}$ phase synchronization, cells were grown in the presence of $2 \mathrm{mM}$ thymidine for $18 \mathrm{~h}$, washed twice with PBS and released into thymidine-free media for 6-8 h, and finally grown again for $12 \mathrm{~h}$ in the presence of $2 \mathrm{mM}$ thymidine. For $\mathrm{M}$ phase synchronization, cells were grown in the presence of $330 \mathrm{nM}$ nocodazole for $18 \mathrm{~h}$. Release from the arrest were accomplished by 2 washes with PBS followed by growth in fresh medium (11).

Construction of Tmubl recombinant lentiviral vectors. The Tmub1 overexpression and knockdown recombinant lentiviral vectors were constructed and purchased from Shanghai GeneChem Co., Ltd. (Shanghai, China). Full length of rat Tmub1 complementary DNA was cloned into GV287 vector (GeneChem, Co., Ltd.) and the resulting vector was designated as flag-Tmub1. The knockdown lentiviral vector GV115 (GeneChem, Shanghai, Co., Ltd.) was constructed with the following shRNA sequence: GGTCTCAACACATACGAC TGA. Negative control lentiviral vectors were constructed in both the overexpression and knockdown lentiviral vectors.

Microarrays and computational analysis. BRL-3A cells were divided into five groups and cultured as follows: Tmub1 overexpression lentivirus-transduced [Lv-Tmub1(+)], Tmub1 knockdown lentivirus-transduced [Lv-Tmub1(-)], Lv-Tmub1(+)-Negative Control, Lv-Tmub1(-)-Negative Control and normal control. The cells were harvested $48 \mathrm{~h}$ post-infection and total RNA for microarray analysis was extracted using an extraction reagent (TRIzol; Invitrogen). Then, complementary DNA was synthesized and labeled before it was purified and hybridized to the microarray (Arraystar, Rockville, MD). The microarray scanning data were extracted using Agilent Feature Extraction software. The quantile normalization and subsequent data processing were performed using the GeneSpring GX v12.1 software package (Agilent Technologies). Overlapped differentially expressed genes with at least a 2.5-fold-change in either direction were considered to be up- or downregulated. Hierarchical clustering was conducted based on differentially expressed
mRNAs using Multiple Experiment Viewer (MeV) v4.6 software. Gene Ontology (GO) (12) and pathway analyses were applied to determine the roles of these differentially expressed mRNAs in the biological GO terms or pathways. The functional analysis of the differentially expressed genes was performed using GO and the KEGG pathway database (13) with the online tool DAVID (http://david.abcc.ncifcrf. gov/home.jsp) (14). The protein interaction network and nodes were determined based on the number of connections between differentially expressed mRNAs using STRING (http://string.embl.de) and the open source software platform Cytoscape_v3.2.1 (National Institute of General Medical Sciences, USA) (15). The microarray data were deposited in NCBI Gene Expression Omnibus and are accessible through GEO series accession number GSE97040.

Reverse transcription-quantitative polymerase chain reaction $(R T-q P C R)$. Total RNA was extracted from the cells using an extraction reagent (TRIzol; Invitrogen; Thermo Fisher Scientific, Inc.). The reverse transcription was performed with PrimeScript $^{\mathrm{TM}}$ RT reagent Kit with gDNA Eraser (Takara Biotechnology Co., Ltd., Dalian, China) according to the manufacturer's instructions, and PCR amplification reactions were performed with SYBR ${ }^{\circledR}$ Premix Ex Taq ${ }^{\mathrm{TM}}$ II (Takara Biotechnology Co., Ltd.) according to the manufacturer's instructions. Primers for qPCR are listed in Table I. The relative quantification of the mRNA levels was normalized to the rat GAPDH (glyceraldehyde-3-phosphate dehydrogenase) levels and calculated with the $\Delta \Delta \mathrm{Cq}$ method (16).

Western blot analysis. Cells were collected and then lysed in 2X SDS (sodium dodecyl sulfate) sample buffer [100 mM Tris-HCl, pH 6.8, 10 mM EDTA (ethylene diamine tetraacetic acid), $4 \%$ SDS, and $10 \%$ glycine] and were then separated by $10 \%$ sodium dodecyl sulfate-polyacrylamide gel electrophoresis, with $30 \mu \mathrm{g}$ of protein in each lane, at $70 \mathrm{~V}$ for $30 \mathrm{~min}$ and then $90 \mathrm{~V}$ for $90 \mathrm{~min}$. Then, the proteins were transferred onto PVDF (polyvinylidene fluoride) membranes for $1.5 \mathrm{~h}$ at $300 \mathrm{~mA}$. After blocking in 5\% non-fat milk for $1 \mathrm{~h}$ at room temperature, the membranes were incubated with the indicated primary antibodies at $4^{\circ} \mathrm{C}$ overnight and horseradish peroxidase-conjugated secondary antibodies at room temperature for $2 \mathrm{~h}$. The GAPDH protein was used as a loading control.

Flow cytometry analysis. After $48 \mathrm{~h}$ of infection, cells were collected and then fixed in $75 \%$ ethanol. Then, the fixed cells were resuspended in propidium iodide/RNase/PBS buffer and incubated in the dark $\left(37^{\circ} \mathrm{C}, 30 \mathrm{~min}\right)$. The cells were then passed through a flow cytometer (FACSCalibur; BD Biosciences, San Diego, CA, USA) equipped with a 488-nm argon laser to measure the DNA content. The data analysis was performed with the appropriate ModFit LT 2.0 software (Verity Software House, Topsham, ME, USA).

Co-immunoprecipitation. First, $10^{7}$ cells were collected and lysed with $500 \mu \mathrm{l}$ of cell lysis buffer (20 mM Tris ( $\mathrm{pH} 7.5)$, $150 \mathrm{mM} \mathrm{NaCl}$ and $1 \%$ Triton X-100 with sodium pyrophosphate, $\beta$-glycerophosphate, EDTA, Na3VO4 and leupeptin) containing 1:50 protease inhibitor cocktail and 1:50 phosphatase inhibitor cocktail. Next, $60 \mu \mathrm{l}$ of SureBeads ${ }^{\mathrm{TM}}$ protein 
Table I. Sequences of primers for RT-qPCR assays.

\begin{tabular}{lll}
\hline Gene & \multicolumn{1}{c}{ Forward primer } & Reverse primer \\
\hline AURKB & 5'-CGGATGCATAATGAGATGGTAGAT-3' & 5'-TCCCCACCATCAGTTCATAGC-3' \\
MCM5 & 5'-GTTCCTGGGAACAGGGTCAC-3' & 5'-CATCTGGGAGCCAGAACCATC-3' \\
INCENP & 5'-GGACTGGAATCGGAGTGGTC-3' & 5'-TCTCCTCAACAACAGCACCC-3' \\
Ns5atp9 & 5'-GCAAAAAGGCATCGGGGAAT-3' & 5'-TCAGGTTGCAAAGGACATGC-3' \\
TTK & 5'-AGGCTGATAAAGAGTCACCACC-3' & 5'-GCTTCTGGGGCCATGTAGTT-3' \\
STAT1 & 5'-AACGGTCCCAAAATGGAGGT-3' & 5'-TGTAGGGCTCAACAGCATGG-3' \\
SERPINE & 5'-GTGGTTCGGCACAATCCAAC-3' & 5'-TGCTGAGTGAAGGCGTAGTG-3' \\
VEGFA & 5'-TTCGTCCAACTTCTGGGCTC-3' & 5'-GCTTTCTGCTCCCCTTCTGT-3' \\
NOS2 & 5'-TGGTGAAAGCGGTGTTCTTTG-3' & 5'-CTTATACTGTTCCATGCAGACAACCTT-3' \\
Pla2g2a & 5'-CATGGCCTTTGGCTCAATTCAGGT-3' & 5'-ACAGTCATGAGTCACACAGCACCA-3' \\
CCNA2 & 5'-GTCAACCCCGAAAAAGTGGC-3' & 5'-GGGGTGATTCAAAACTACCATCC-3' \\
Rrm2 & 5'-TTTGTCCCCTTGCCATTA-3' & 5'-GCAGTGACCATCAAGCAAG-3' \\
Sirt1 & 5'GAATTCTTAACCAGCATTGGGAACTTTAGC-3' & 5'GGATCCTTGGAGGAAGATAATCCAGTCA-3' \\
MCM3 & 5'-TGTCTCGGTTTGACCTGCTC-3' & 5'-TCCAGTGTCCGTGCTGTAAC-3' \\
PLK4 & 5'-AGGGAAGCTAGGCACTTCATG-3' & 5'-GGAAGACCACCTTTTGAC-3' \\
GAPDH & 5'-GCCATCAACGACCCCTTCATT-3' & 5'-CGCCTGCTTCACCACCTTCTT-3' \\
\hline
\end{tabular}

G magnetic beads (no. 1614023; Bio-Rad Laboratories, Inc., Hercules, CA, USA) were incubated with $3 \mu \mathrm{g}$ of antibody on a rotating platform for $30 \mathrm{~min}$ at room temperature and then incubated with $500 \mu \mathrm{l}$ of $(1 \mathrm{mg})$ cell lysate and rotated overnight at $4^{\circ} \mathrm{C}$. The IP products were eluted by $40 \mu \mathrm{l}$ of $1 \mathrm{X}$ Laemmli buffer and incubated for $10 \mathrm{~min}$ at $95^{\circ} \mathrm{C}$. Western blot analysis was used for the subsequent protein detection.

5-Ethynyl-20-deoxyuridine (EdU) assays. BRL-3A cells were seeded onto 24-well plates. Twenty-four h later, the BRL-3A cells were infected with either Lv-Tmub1 (+), Lv-Tmub1 (-) or NC vectors accordingly. Forty-eight h after infection, the cell proliferation was determined in vitro via the EdU DNA Proliferation in Detection kit (Nanjing KeyGen Biotech Co., Ltd., Nanjing, China) based on the manufacturer's instructions.

Cell Counting Kit-8 (CCK-8) assay. Cells were seeded at a concentration of $10^{3} / \mathrm{ml}$ with 5 replicates in a 96-well plate and cultured overnight. On the following day, the cell viability was measured by the CCK-8 (Dojindo Molecular Technologies, Inc., Kumamoto, Japan). A volume of $10 \mu 1$ of CCK- 8 solution was added to each well at $0,24,48$, or $72 \mathrm{~h}$ after culture. The cells were incubated at $37^{\circ} \mathrm{C}$ for $2 \mathrm{~h}$, and the absorbance values at $450 \mathrm{~nm}$ were measured using an enzyme-linked analyzer (Thermo Fisher Scientific, Inc.).

Statistical analysis. All experimental data were analyzed by Graphad Prism 5.1 (GraphPad Software, Inc., La Jolla, CA, USA) or SPSS version 19 (IBM Corp., Armonk, NY, USA). Data were presented as the mean \pm SD of three independent experiments. Statistical analyses shown in the figures were performed using t-tests or one-way analysis of variance with least significant difference post hoc tests. All graphs were plotted by the use of Graphad Prism 5.1 (GraphPad Software, Inc.). $\mathrm{P}<0.05$ was considered to indicate a statistically significant difference.

\section{Results}

Transcriptional profiling in Tmubl overexpressed or knockdown BRL-3A cells. We analyzed the mRNA expression profiles of cells infected with lentivirus either overexpressing or knocking down Tmub1 and of normal control BRL-3A cells (Tmub1 expression were shown in Fig. 1D). The microarray analysis identified 836 differentially expressed genes that were either up- or downregulated, and 127 node genes were screened by STRING. The GO and KEGG pathway analysis using the DAVID database demonstrated that the top five regulated GO categories targeted by Tmub1 overexpression and knockdown were response to cellular process, biological regulation, regulation of biological process, response to stimulus, and regulation of cellular process. The most significant pathway of the differentially expressed genes was cell cycle pathway (Fig. 1C). The node gene network was screened by the number of interaction edges by Cytoscape software (Fig. 1B), and the clustering analysis showed distinct trends in the expression of node genes and key node genes among the 5 groups (Fig. 1A). Seventeen key node genes were identified, and RT-qPCR analysis confirmed the microarray data (Fig. 1E). These data demonstrated the close relation among Tmubl and the cell cycle related genes.

Tmubl is a negative regulator of the cell cycle and proliferation in hepatocyte cells. In order to investigate whether Tmub1 influences cell proliferation in BRL-3A cells, we conducted EdU and CCK-8 assays. The results showed that, compared to the normal control group, The cell proliferation rate of Lv-Tmub1 (-) cells was significantly higher and the cell proliferation rate of Lv-Tmub1 (+) cells was significantly lower (Fig. 2A-C). These findings demonstrated that Tmub1 has a negative impact on the BRL-3A cell proliferation.

In order to investigate how the cell cycle pathway was affected by Tmub1 expression, cell cycle analysis were 
A

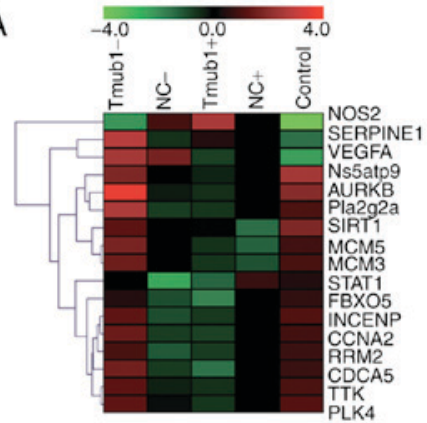

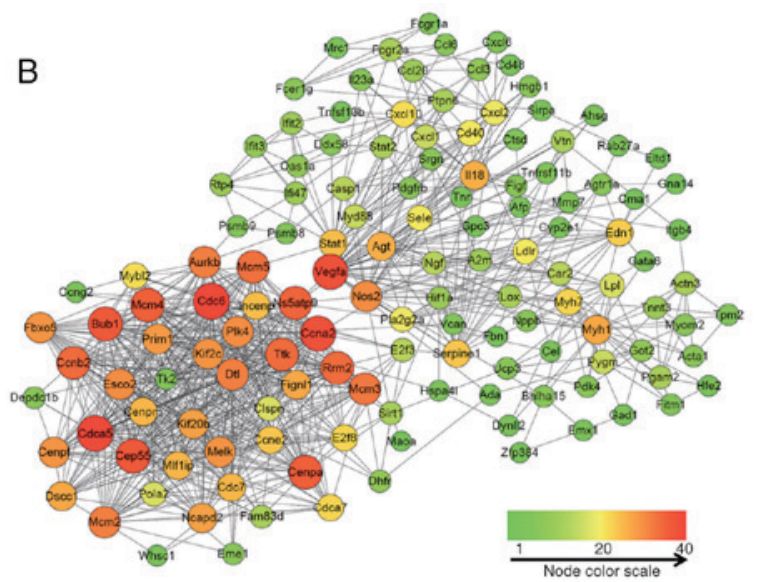

D Tmub1
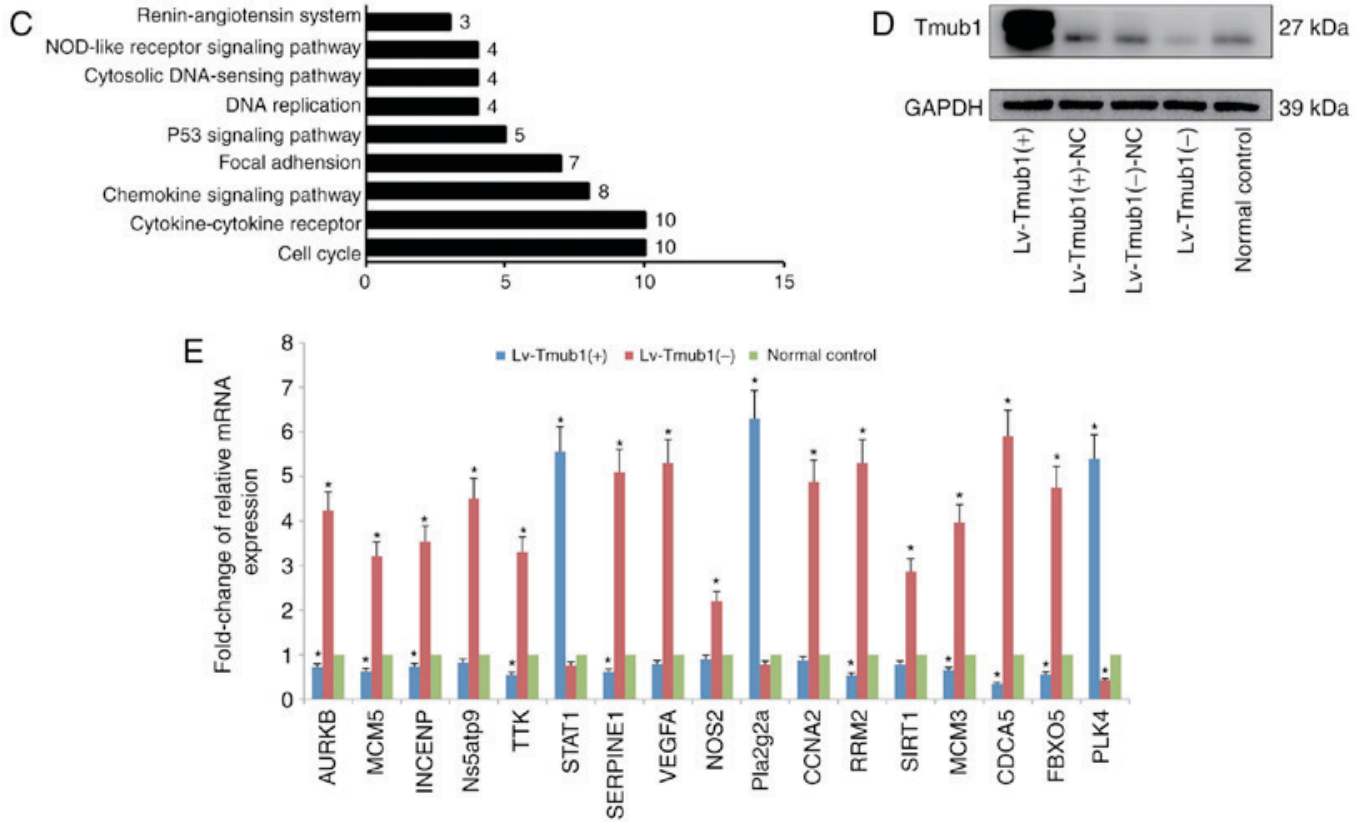

Figure 1. Differentially expressed genes after Tmub1 overexpression or knockdown. (A) Hierarchical clustering of Tmub1-, NC-, Tmub1+, NC+ and control BRL-3A cells (columns) and 17 key node genes (rows). Up-regulated genes were marked in red and down-regulated genes were marked in green. (B) Network of node genes. The differentially expressed genes after Tmub1 overexpression or knockdown were subjected to STRING (http://string.embl.de) to screen the node genes, network of node genes was demonstrated by software Cytoscape v3.2.1. The color brightness and shape size of nodes were determined by the number of interaction edges. (C) Counts of diffident genes in KEGG pathways analysis by the DAVID database. (D) Tmub1 protein expression by Western blotting assay. Cell lysates were collected 2 days after lentivirus vector infection. (E) Reverse transcription-quantitative polymerase chain reaction validation of 17 key node genes. The results were normalized to the GAPDH values for each gene, samples were normalized to the normal control. The fold-changes were shown as mean \pm standard deviation in three independent experiments. Compared with control group, statistically significant differences were determined by one-way analysis of variance with least significant difference post hoc test, indicated as: ${ }^{*} \mathrm{P}<0.05$ vs. the normal control. Tmub1, transmembrane and ubiquitin-like domain containing protein 1; NC, normal control; KEGG, Kyoto Encyclopedia of Genes and Genomes; DAVID, Database for Annotation, Visualization, and Integrated Discovery.

conducted. The proportion cells in S phase of the Lv-Tmub1 (-) group increased significantly compared to that of the Lv-Tmub1 (+) and normal control groups, while the proportion of cells in G1 phase decreased significantly; the proportion of Lv-Tmub1 (+) cells in G1 phase increased significantly, while the proportion of cells in $\mathrm{S}$ phase decreased significantly (Fig. 2D and E). These results indicated that Tmub1 may affect the $\mathrm{G} 1 / \mathrm{S}$ phase transition.

Tmubl interacts with cyclin A2 in the cell cycle. Cyclin A plays important roles in the G1/S and G2/M transitions. Cyclin A-CDK2 complexes are active through $\mathrm{S}$ phase, while cyclin A-CDC2 and cyclin B-CDC2 complexes are active during the $\mathrm{G} 2$ and $\mathrm{M}$ phases (17). Cyclin A and cyclin B1 must be degraded in $\mathrm{M}$ phase for a proper G2/M transition (18). To investigate the possible relationship between Tmub1 and cell cycle cyclins in M phase, Lv-Tmub1 (+), Lv-Tmub1(-) and normal control BRL-3A cells were synchronized in M phase by nocodazole treatment (Fig. 3A, the synchronization efficiency of all cell groups were shown in Fig. 3B) and then lysed $0,0.5,1,1.5 \mathrm{~h}$ after being released from the arrest. The expression of cyclin A2, cyclin B1, cyclin D1 and cyclin E1 was detected by Western blot analysis. In Tmub1-overexpressing BRL-3A cells, the level of cyclin A2 remained high at $1 \mathrm{~h}$, while the cyclin A2 level in the negative control cells significantly dropped at $1 \mathrm{~h}$. In contrast, the cyclin A2 level in the 
A
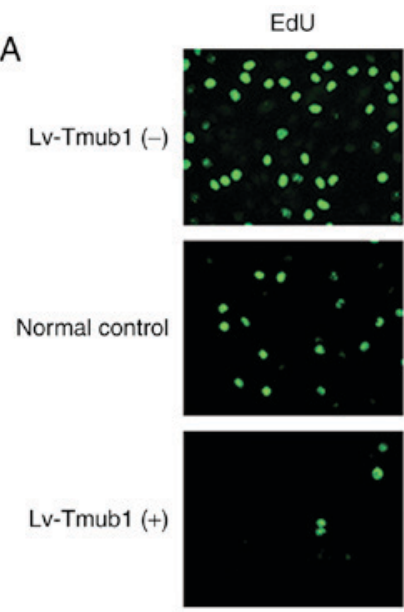

B
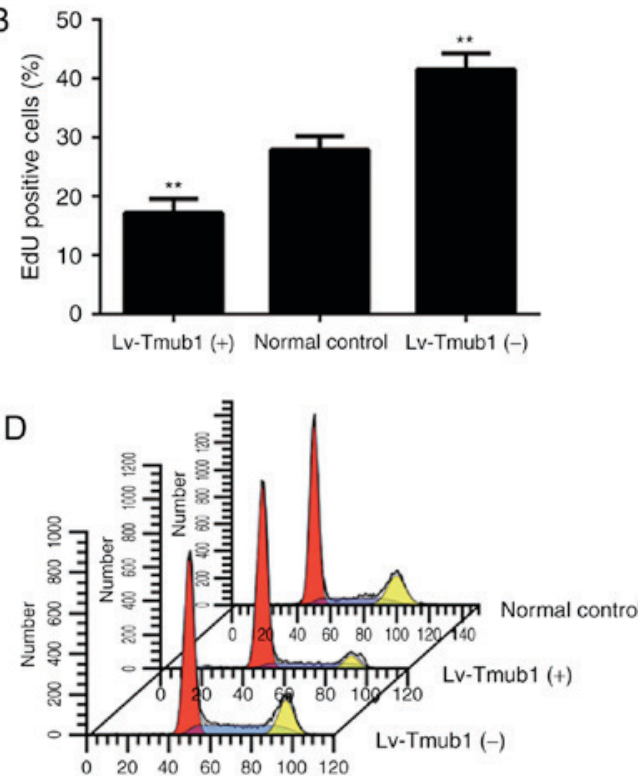

Hoechst
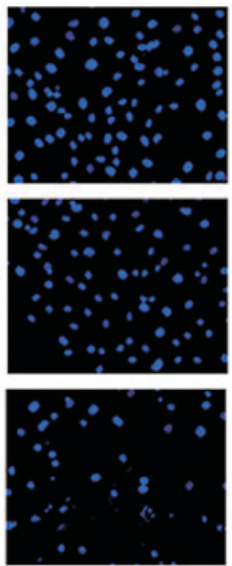

Merge
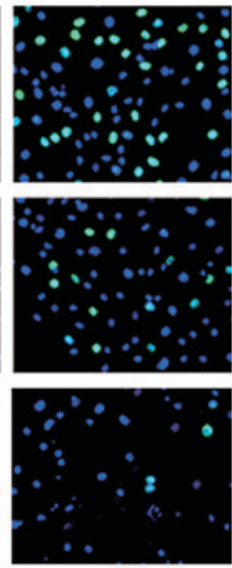

C

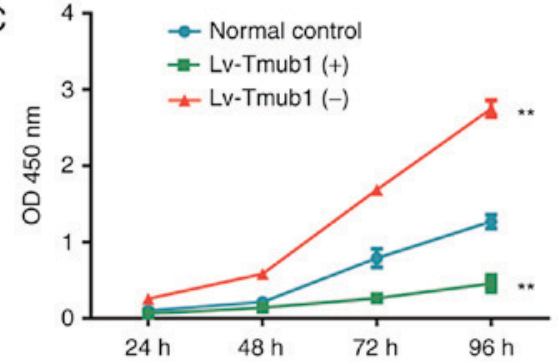

E

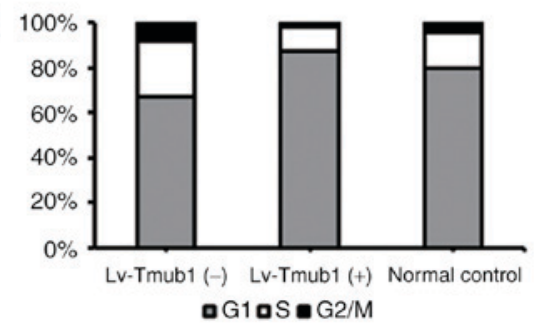

Figure 2. Tmub1 inhibits BRL-3A cell proliferation and the cell cycle progression. (A and B) Effects of Tmub1 on cell proliferation in Tmub1+, Tmub1- and normal control BRL-3A cells by EdU assay and (C) Cell Counting Kit- 8 assay. Values represent the mean \pm standard deviation of three separate experiments. Compared with control group, statistically significant differences were determined by one-way analysis of variance with least significant difference post hoc test, indicated as: ${ }^{* *} \mathrm{P}<0.01$ vs. the normal control. (D and E) Detection of cell cycle in Tmub1+, Tmub1- and normal control BRL-3A cells by flow cytometry. Distribution of cells in G0/G1, S and G2/M phases were calculated. Tmub1, transmembrane and ubiquitin-like domain containing protein 1; OD, optical density; EdU, 5-Ethynyl-20-deoxyuridine.

Tmub1-knockdown BRL-3A cells dropped even earlier. The same result was observed for cyclin $\mathrm{B} 1$, which indicated that Tmub1 may inhibit cyclin A2 and cyclin B1 degradation in $\mathrm{M}$ phase (Fig. 3C, upper panel). The expression patterns of cyclin D1 and cyclin E1 showed no difference between the three groups, However, the results showed that the expression of cyclin D1 and cyclin E1 was negatively associated with that of Tmub1 (Fig. 3C, lower panel).

Next, we conducted co-immunoprecipitation assays to examine the possible interaction between Tmub1 and cyclins A2, B1, D1 and E1. Interestingly, Only cyclin A2 showed the possible interaction with Tmub1 (Fig. 4B). Further cell cycle synchronization and co-immunoprecipitation assays were conducted to explore the phases in which Tmub1 and cyclin A2 specifically interact. As shown in Fig. 4C-E, Tmub1 bound to cyclin A2 in G1, S and M phases. These results indicated that the interaction between Tmub1 and cyclin A2 may be close throughout the cell cycle, Tmubl may interact with
Cyclin A 2 for both G1/S transition and M phase progression. The results in Fig. 4A show that cyclin A2 expression was significantly lower in the Tmub1-knockdown BRL-3A cells, indicating that Tmub1 may function as a positive regulator of cyclin A2 during the cell cycle.

\section{Discussion}

Tmub1 was first described as a hepatocyte shuttling protein that is ubiquitously expressed and moves between the nucleus and cytoplasm. However, the studies on Tmub1 in the past decade since its discovery are scattered and the main biological function of Tmub1 still has not been revealed yet. Within the nucleus of resting cells, Tmub1 overexpression causes cell cycle arrest in G0/G1, and Tmub1 knockdown causes centrosome hyperamplification, leading to multinucleated cells and the formation of micronuclei (5). Recent studies have indicated that the Tmub1 gene was overexpressed in cultured primary 
A<smiles>CC=C(C)C</smiles>
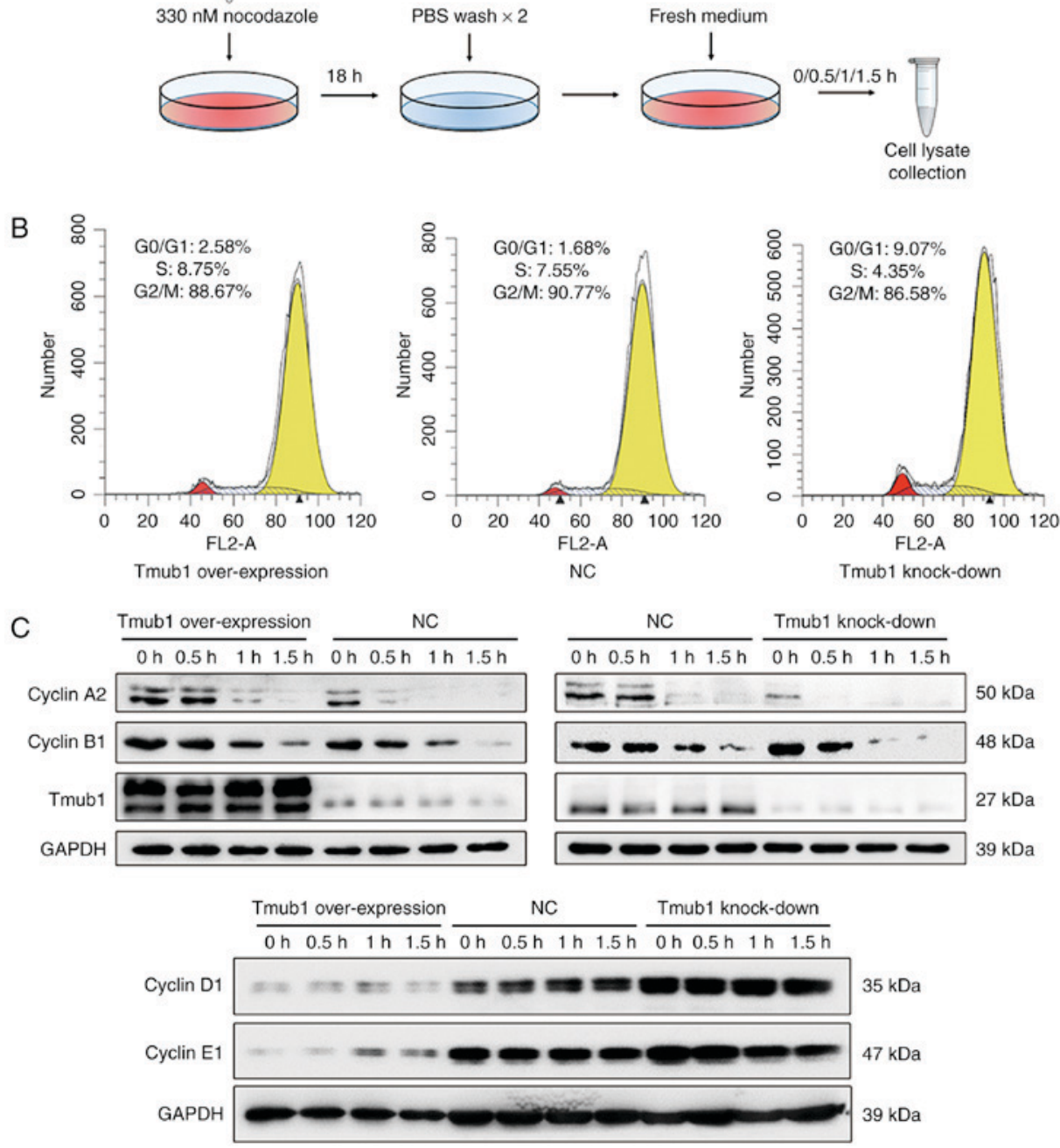

Figure 3. Tmub1 influences cyclins expression in M phase. (A) M phase synchronization method by nocodazole. Tmub1+, Tmub1- and NC BRL-3A cells were synchronized in M phase by $330 \mathrm{nM}$ nocodazole treatment for $18 \mathrm{~h}$, followed by replacement of fresh medium. Cell were harvest every $30 \mathrm{~min}$ for the following Western Blot analysis. (B) M phase synchronization efficiency by flow cytometry analysis. Tmub1+, Tmub1- and NC BRL-3A cells were collected at $0 \mathrm{~h}$ after the nocodazole treatment. (C) Cell cycle cyclins were detected by western blot analysis. GAPDH was used as the loading control. Tmub1, transmembrane and ubiquitin-like domain containing protein 1; NC, normal control.

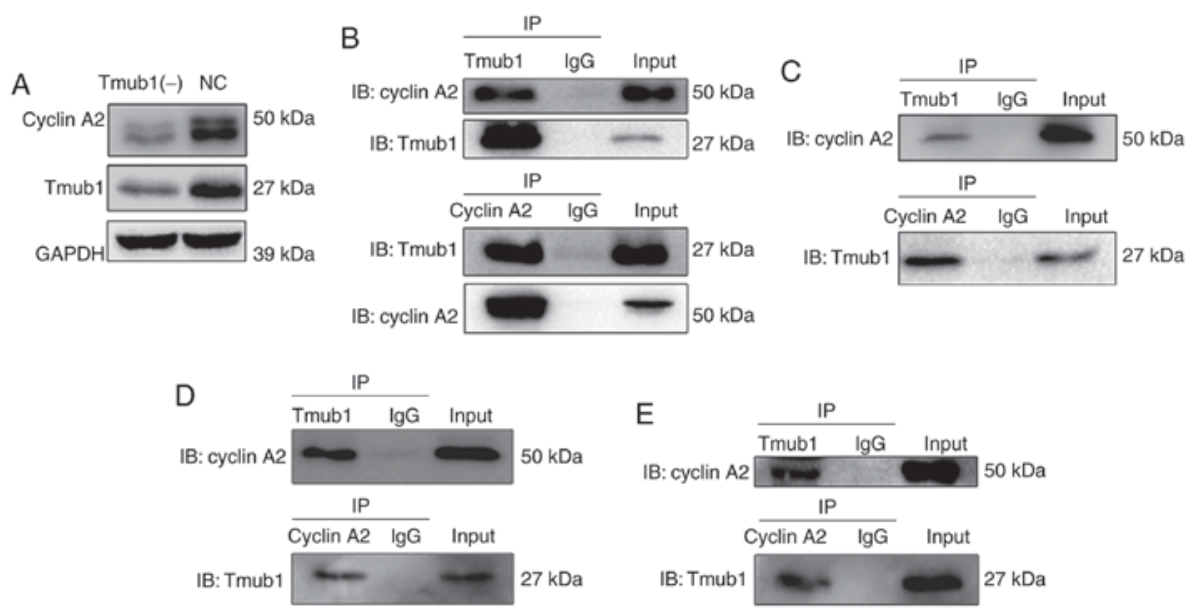

Figure 4. Tmub1 interacts with cyclin A2 in the cell cycle. (A) Tmub1- and NC-BRL-3A cell lysates were subjected to western blot analysis for cyclin A2, Tmub1 and GAPDH. (B) Co-immunoprecipitation revealed the interaction between the endogenous Tmub1 and cyclin A2 in rat normal BRL-3A cells. (C-E) Co-immunoprecipitation of Tmub1 and cyclin A2 in the cell cycle. BRL-3A cells were synchronized in (C) G1 phase, (D) S phase or (E) M phase by serum starvation, double thymidine block or nocodazole treatment, respectively. Tmub1, transmembrane and ubiquitin-like domain containing protein 1; IP, immunoprecipitation. 
neurons (9). Tmub1 acts as a bridge in the NPM and p19Arf interaction, indicating the possible ability to oppose tumor cell proliferation (4). Our previous study shows that IL-6 is the upstream regulator of Tmub1, and Tmub1 knockdown synergizes with IL-6 in inducing hepatocyte proliferation (8). Moreover, $\mathrm{C} / \mathrm{EBP} \beta$ is a key transcription factor involved in the regulation of Tmub1 expression (19). To investigate the role of Tmub1 in the cell cycle, whether Tmub1 functions as a key transcriptional regulatory molecule of the cell cycle-related genes is the first question to be answered.

In this study, stable liver cells (Tmub1 gene overexpression or knockdown) were used to study the influence of Tmub1 expression on the genome-wide transcriptional profile by mRNA microarray analysis. By comparing these gene expression profiles to that of the normal rat liver cell line BRL-3A, 836 differentially expressed genes (up- or downregulated) with 127 node genes were identified. These data demonstrated that overexpression or knockdown of Tmub1 may affect the expression of many genes with important functions in regenerating liver cells. Further pathway analysis identified pathways with important roles in the regulation of cellular proliferation and the cell cycle (20-24). Among the node genes, 17 key node genes (AURKB, MCM5, INCENP, Ns5atp9, TTK, STAT1, SERPINE1, VEGFA, NOS2, Pla2g2a, CCNA2, RRM2, SIRT1, MCM3, CDCA5, FBXO5, and PLK4) were screened and validated by RT-qPCR, and because most of these genes are related to the cell cycle (25-30), Tmub1 may be a crucial regulatory protein in the cell cycle-regulating network.

Many proteins regulating cell cycle transitions and progression through checkpoints have been studied in the past few decades (31). Among these proteins, cyclin-CDK complexes are basic regulators of cell cycle progression (32). Cyclins A and B play a central role in the control of mitosis, with cyclin A being degraded in prometaphase before cyclin B in metaphase by the ubiquitin-proteasome-system or autophagy (33). Mammalian cells have two types of cyclins As; cyclin A1 is specifically expressed in the testis, while cyclin A2 ubiquitously expressed. Accordingly, cyclin A2 is usually linked to cell proliferation and as such is often found expressed at a high level in human cancers (34). Cyclin A also mediates the progression through S phase by forming the CDK2-cyclin A complex (35). P21 binds and inactivates cyclin-CDK complexes that mediate G1/S progression, resulting in the lack of phosphorylation of $\mathrm{Rb}, \mathrm{E} 2 \mathrm{~F}$ sequestration and cell cycle arrest at the G1/S transition (36). Our findings indicated that Tmub1 may participate in the G1/S transition and $\mathrm{S}$ phase progression by interacting with cyclin A2 and may delay cyclin A2 and cyclin B degradation in $\mathrm{M}$ phase, which strongly suggested that Tmub1 is a cell cycle-associated protein.

In addition to the canonical ubiquitylation pathway, proteins can also be modified through attachment to ubiquitin-like proteins (UBLs), which have conserved ubiquitin-like sequences and control different types of biological processes $(37,38)$. Although UBLs are found to have diverse roles in various processes, the studies on the functions of many members in this family are only beginning. As a ubiquitin-like protein, Tmub1 was found to mediate the ubiquitylation and degradation of the HMG-CoA reductase HMGCR (39). In this process, Tmub1 bridges SPFH2 to a membrane-bound ubiquitin ligase gp78 in endoplasmic reticulum membranes.
Our results showed that Tmub1 may inhibit the degradation of cyclin A2 and B1, indicating that Tmub1 may play different roles in ubiquitylation by interacting with different proteins. Therefore, the question of how Tmubl functions as an ubiquitin-like protein remains to be answered. The UBL domain of Tmub1 may contribute to the posttranslational modification of several cell cycle proteins, and Tmub1 may function as an 'effector' in the complicated and precise network of cell cycle regulation. In further studies, we intend to investigate how Tmub1 regulates cell cycle proteins, specifically focusing on the interaction between Tmub1 and cyclin A2, and the possible regulatory role of Tmubl in cyclin ubiquitylation and degradation.

In conclusion, our study identified Tmub1 as a cell cycle-associated protein. Tmub1 regulates gene expression, inhibits hepatocyte proliferation and affects the cell cycle by interacting with cyclins.

\section{Acknowledgements}

This study was supported by the National Natural Science Foundation of China (grant number: 81270523).

\section{References}

1. Riehle KJ, Dan YY, Campbell JS and Fausto N: New concepts in liver regeneration. J Gastroenterol Hepatol 26 (Suppl 1): S203-S212, 2011

2. Fausto N, Campbell JS and Riehle KJ: Liver regeneration. J Hepatol 57: 692-694, 2012.

3. Della Fazia MA, Castelli M, Bartoli D, Pieroni S, Pettirossi V, Piobbico D, Viola-Magni M and Servillo G: HOPS: A novel cAMP-dependent shuttling protein involved in protein synthesis regulation. J Cell Sci 118: 3185-3194, 2005.

4. Castelli M, Pieroni S, Brunacci C, Piobbico D, Bartoli D, Bellet MM, Colombo E, Pelicci PG, Della Fazia MA and Servillo G: Hepatocyte odd protein shuttling (HOPS) is a bridging protein in the nucleophosmin-p19 Arf network. Oncogene 32: 3350-3358, 2013.

5. Pieroni S, Della Fazia MA, Castelli M, Piobbico D, Bartoli D, Brunacci C, Bellet MM, Viola-Magni M and Servillo G: HOPS is an essential constituent of centrosome assembly. Cell Cycle 7: 1462-1466, 2008.

6. Herrmann J, Lerman LO and Lerman A: Ubiquitin and ubiquitin-like proteins in protein regulation. Circ Res 100: 1276-1291, 2007.

7. Eifler K and Vertegaal AC: Mapping the SUMOylated landscape. FEBS J 282: 3669-3680, 2015.

8. Liu M, Liu H, Wang X, Chen P and Chen H: IL-6 induction of hepatocyte proliferation through the Tmub1-regulated gene pathway. Int J Mol Med 29: 1106-1112, 2012.

9. Zhang W, Savelieva KV, Suwanichkul A, Small DL, Kirkpatrick LL, Xu N, Lanthorn TH and Ye GL: Transmembrane and ubiquitin-like domain containing 1 (Tmub1) regulates locomotor activity and wakefulness in mice and interacts with CAMLG. PLoS One 5: e11261, 2010.

10. Yang H, Takagi H, Konishi Y, Ageta H, Ikegami K, Yao I, Sato S, Hatanaka K, Inokuchi K, Seog DH and Setou M: Transmembrane and ubiquitin-like domain-containing protein 1 (Tmub1/HOPS) facilitates surface expression of GluR2-containing AMPA receptors. PLoS One 3: e2809, 2008.

11. Cedeno C,La Monaca E, Esposito M and Gutierrez GJ: Detection and analysis of cell cycle-associated APC/C-mediated cellular ubiquitylation in vitro and in vivo. Methods Mol Biol 1449: 251-265, 2016.

12. Ashburner M, Ball CA, Blake JA, Botstein D, Butler H, Cherry JM, Davis AP, Dolinski K, Dwight SS, Eppig JT, et al: Gene ontology: Tool for the unification of biology. The gene ontology consortium. Nat Genet 25: 25-29, 2000.

13. Kanehisa M and Goto S: KEGG: Kyoto encyclopedia of genes and genomes. Nucleic Acids Res 28: 27-30, 2000. 
14. Huang DW, Sherman BT and Lempicki RA Bioinformatics enrichment tools: Paths toward the comprehensive functional analysis of large gene lists. Nucleic Acids Res 37: $1-13,2009$

15. Shannon P, Markiel A, Ozier O, Baliga NS, Wang JT, Ramage D, Amin N, Schwikowski B and Ideker T: Cytoscape: A software environment for integrated models of biomolecular interaction networks. Genome Res 13: 2498-2504, 2003.

16. Livak KJ and Schmittgen TD: Analysis of relative gene expression data using real-time quantitative PCR and the 2(-Delta Delta C(T)) method. Methods 25: 402-408, 2001.

17. Coller HA: What's taking so long? S-phase entry from quiescence versus proliferation. Nat Rev Mol Cell Biol 8: 667-670, 2007.

18. van Leuken R, Clijsters L and Wolthuis R: To cell cycle, swing the APC/C. Biochim Biophys Acta 1786: 49-59, 2008.

19. Liu M, Yuan T, Liu H and Chen P: CCAAT/enhancer-binding protein $\beta$ regulates interleukin- 6 -induced transmembrane and ubiquitin-like domain containing 1 gene expression in hepatocytes. Mol Med Rep 10: 2177-2183, 2014.

20. Vansaun MN, Mendonsa AM and Lee Gorden D: Hepatocellular proliferation correlates with inflammatory cell and cytokine changes in a murine model of nonalchoholic fatty liver disease. PLoS One 8: e73054, 2013.

21. Chiu YS, Wei CC, Lin YJ, Hsu YH and Chang MS: IL-20 and IL-20R1 antibodies protect against liver fibrosis. Hepatology 60: 1003-1014, 2014

22. Liu W, Li X, Chu ES, Go MY, Xu L, Zhao G, Li L, Dai N, Si J, Tao Q, et al: Paired box gene 5 is a novel tumor suppressor in hepatocellular carcinoma through interaction with p53 signaling pathway. Hepatology 53: 843-853, 2011.

23. Mirzayans R, Pollock S, Scott A, Gao CQ and Murray D: Metabolic labeling of human cells with tritiated nucleosides results in activation of the ATM-dependent $\mathrm{p} 53$ signaling pathway and acceleration of DNA repair. Oncogene 22: 5562-5571, 2003.

24. Marra F and Tacke F: Roles for chemokines in liver disease. Gastroenterology 147: 577-594.e1, 2014.

25. Malumbres $M$ and Barbacid M: Cell cycle, CDKs and cancer: A changing paradigm. Nat Rev Cancer 9: 153-166, 2009.

26. Magatti M, De Munari S, Vertua E and Parolini O: Amniotic membrane-derived cells inhibit proliferation of cancer cell lines by inducing cell cycle arrest. J Cell Mol Med 16: 2208-2218, 2012.

27. Uren AG, Wong L, Pakusch M, Fowler KJ, Burrows FJ, Vaux DL and Choo KH: Survivin and the inner centromere protein INCENP show similar cell-cycle localization and gene knockout phenotype. Curr Biol 10: 1319-1328, 2000.
28. Li K, Ma Q, Shi L, Dang C, Hong Y, Wang Q, Li Y, Fan W, Zhang $L$ and Cheng J: NS5ATP9 gene regulated by NF-kappaB signal pathway. Arch Biochem Biophys 479: 15-19, 2008.

29. Terme M, Pernot S, Marcheteau E, Sandoval F, Benhamouda N, Colussi O, Dubreuil O, Carpentier AF, Tartour E and Taieb J: VEGFA-VEGFR pathway blockade inhibits tumor-induced regulatory T-cell proliferation in colorectal cancer. Cancer Res 73: 539-549, 2013.

30. Cho RJ, Huang M, Campbell MJ, Dong H, Steinmetz L, Sapinoso L, Hampton G, Elledge SJ, Davis RW and Lockhart DJ: Transcriptional regulation and function during the human cell cycle. Nat Genet 27: 48-54, 2001

31. Mukherji M, Bell R, Supekova L, Wang Y, Orth AP, Batalov S, Miraglia L, Huesken D, Lange J, Martin C, et al: Genome-wide functional analysis of human cell-cycle regulators. Proc Natl Acad Sci USA 103: 14819-14824, 2006

32. Bloom J and Cross FR: Multiple levels of cyclin specificity in cell-cycle control. Nat Rev Mol Cell Biol 8: 149-160, 2007.

33. Loukil A, Zonca M and Rebouissou C: High-resolution live-cell imaging reveals novel cyclin A2 degradation foci involving autophagy. J Cell Sci 127: 2145-2150, 2014.

34. Bendris N, Loukil A, Cheung C, Arsic N, Rebouissou C, Hipskind R, Peter M, Lemmers B and Blanchard JM: Cyclin A2: A genuine cell cycle regulator? Biomol Concepts 3: 535-543, 2012.

35. Lee RS, Sohn S, Shin KH, Kang MK, Park NH and Kim RH: Bisphosphonate inhibits the expression of cyclin A2 at the transcriptional level in normal human oral keratinocytes. Int $\mathrm{J}$ Mol Med 40: 623-630, 2017

36. Besson A, Dowdy SF and Roberts JM: CDK inhibitors: Cell cycle regulators and beyond. Dev Cell 14: 159-169, 2008.

37. Hochstrasser M: Origin and function of ubiquitin-like proteins. Nature 458: 422-429, 2009

38. Kerscher O, Felberbaum R and Hochstrasser M: Modification of proteins by ubiquitin and ubiquitin-like proteins. Annu Rev Cell Dev Biol 22: 159-180, 2006.

39. Jo Y, Sguigna PV and DeBose-Boyd RA: Membrane-associated ubiquitin ligase complex containing gp78 mediates sterol-accelerated degradation of 3-hydroxy-3-methylglutaryl-coenzyme A reductase. J Biol Chem 286: 15022-15031, 2011.

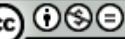

This work is licensed under a Creative Commons Attribution-NonCommercial-NoDerivatives 4.0 International (CC BY-NC-ND 4.0) License. 